\title{
Complicações materno-fetais em gestações gemelares: uma revisão integrativa
}

\author{
Fetal maternal complications in twin pregnancies: an integrative review
}

Complicaciones maternas fetales en embarazos gemelares: una revisión integradora

Daniella Xavier de Souza Cerqueira Caçapava ${ }^{1 *}$, Amanda Rezende de Medeiros ${ }^{1}$, Layanna Nayra dos Santos ${ }^{1}$, Murillo Santos da Cruz Vieira ${ }^{1}$, Nathália Carolinne Rabêlo de Souza ${ }^{1}$, Marcela de Andrade Silvestre ${ }^{1}$, Ana Karina Marques Salge Mendonça².

\section{RESUMO}

Objetivo: Identificar as complicações em gestações gemelares e relacioná-las com as consequências materno-fetais. Métodos: Trata-se de uma revisão integrativa da leitura de artigos nas bases Scientific Electronic Library Online, Literatura Latino-Americana do Caribe em Ciências da Saúde, Turning Research Into Practice e Google Acadêmico, que inclui as produções dos últimos cinco anos. A busca foi realizada com os seguintes descritores de saúde: "Twin Pregnancy and Complications", "Gravidez de gêmeos and complicações" e "Pregnancy Complications and Twinning". A seleção dos artigos foi feita em três etapas, com aplicação de filtros, leitura do título e resumo e leitura dos artigos. Resultados: Foram selecionados 20 artigos e identificadas duas categorias de complicações, sendo elas maternas e fetais. Quanto às complicações maternas houve uma prevalência de parto prematuro, ruptura prematura das membranas, distúrbios hipertensivos, eclampsia e pré-eclampsia, e quanto às fetais, internação, morte fetal e baixo peso. Considerações finais: A análise das produções científicas encontradas nos permitiu concluir que há uma maior prevalência de complicações maternas e fetais em gestações gemelares comparado com gestações únicas. Embora estejam separadas didaticamente em categorias, a maioria das complicações se relacionam, sendo algumas associações entre causa e consequência.

Palavras-chave: Gravidez de gêmeos, Complicações, Complicações na gravidez.

\section{ABSTRACT}

Objective: Recognize the complications in twin pregnancies and relate them to the maternal-fetal consequences. Methods: This is an integrative review based on the reading of articles found in the Scientific Electronic Library Online, Literatura Latino-Americana do Caribe em Ciências da Saúde, Turning Research Into Practice and Google Scholar databases, which includes the productions of the last five years. The search was carried out with the following health descriptors: "Twin Pregnancy and Complications", "Pregnancy of twins and complications" and "Pregnancy Complications and Twinning". The selection of articles was done in three stages, with the application of filters, reading the title and summary and reading the articles. Results: 20 articles were selected and two categories of complications were identified, maternal and fetal. As for maternal complications, there was a prevalence of premature birth, premature rupture of the membranes, hypertensive disorders, eclampsia and pre-eclampsia, and for the fetal ones, hospitalization, fetal death and low weight. Final considerations: The analysis of the articles found allowed us to conclude that there is a higher prevalence of maternal and fetal complications in twin pregnancies compared to single pregnancies. Although they are didactically separated into categories, most complications are related, with some associations between cause and consequence.

Keywords: Twin pregnancy, Complications, Pregnancy complications.

${ }^{1}$ Centro Universitário de Anápolis - UniEvangélica, Anápolis - GO *E-mail: daniellaxavier0101@gmail.com 2Universidade Federal de Goiás, Goiânia - GO. 


\section{RESUMEN}

Objetivo: Identificar complicaciones en embarazos gemelares y relacionarlas con las consecuencias materno-fetales. Métodos: Se trata de una revisión integradora de la lectura de artículos en las bases Scientific Electronic Library Online, Literatura Latinoamericana de el Caribe en Ciencias de la Salud, Turning Research Into Practice y Google Académico, que incluye las producciones de los últimos cinco años. La búsqueda se realizó con los siguientes descriptores de salud: "Embarazo de gemelos y complicaciones", "Embarazo de gemelos y complicaciones" y "Complicaciones del embarazo y hermanamiento". La selección de artículos se realizó en tres etapas, con aplicación de filtros, lectura del título y resumen y lectura de los artículos. Resultados: Se seleccionaron 20 artículos y se identificaron dos categorías de complicaciones, maternas y fetales. En cuanto a las complicaciones maternas, hubo prevalencia de parto prematuro, rotura prematura de membranas, trastornos hipertensivos, eclampsia y preeclampsia, y para las fetales, hospitalización, muerte fetal y bajo peso. Consideraciones finales: El análisis de las producciones científicas encontradas permitió concluir que existe una mayor prevalencia de complicaciones maternas y fetales en los embarazos gemelares en comparación con los embarazos únicos. Están divididos didácticamente en categorías, la mayoría de las complicaciones están relacionadas, con algunas asociaciones entre causa y consecuencia.

Palabras clave: Embarazo gemelar, Complicaciones, Complicaciones del embarazo.

\section{INTRODUÇÃO}

A gravidez gemelar consiste na presença simultânea de dois ou mais fetos por gestação, dentro do útero ou fora dele, sendo caracterizada em dupla, tripla e múltipla de elevada ordem: quádrupla, quíntupla, sêxtupla e assim por diante. Ademais, as principais complicações são o parto pré-termo, a insuficiência placentária, oligodramnia e a síndrome da transfusão feto-fetal, que por sua vez ocasiona polidramnia no outro gemelar e a elevada morbimortalidade fetal e neonatal. Nesse sentido, outro aspecto relevante é que cerca de $50 \%$ das gestações identificadas no primeiro trimestre completam o processo e resultam em parto gemelar, isso acontece devido a complicações que podem se manifestar durante o percurso (REZENDE JF; MONTENEGRO CA, 2014).

A prevalência da gestação múltipla espontânea é aproximadamente de 1-2\%. Nas últimas décadas, nos países desenvolvidos, houve uma elevação para 3-4\%, em decorrência do aumento da idade materna e da utilização de técnicas de reprodução assistida. As gestações gemelares, comparadas com as únicas, expressam maior risco de complicações fetais como defeitos congênitos, partos pré-termo, restrito crescimento intrauterino, paralisia cerebral e mortalidade perinatal. Somado a isso, existe maior incidência de complicações maternas relacionadas a transtornos hipertensivos, diabetes gestacional, parto cesáreo e hemorragia pós-parto. A corionicidade é um fator importante para um pré-natal bem sucedido em casos de gestações gemelares (SOCIEDADE ESPANHOLA DE GINECOLOGIA E OBSTETRÍCIA, 2016).

Nesse diapasão, os gêmeos ainda podem ser monozigótico (MZ) ou dizigótico (DZ). Os MZ, univitelinos, uniovulares ou gêmeos verdadeiros, resultam da fertilização de um óvulo por um único espermatozoide e representam cerca de $1 / 3$ dos casos. Além disso, podem corresponder a placentação monocoriônica (MC) ou dicoriônica (DC), mas obrigatoriamente o sexo é igual, pertencem aos mesmos grupos sanguíneos, as características físicas e as tendências patológicas são semelhantes. Os gêmeos DZ, ao contrário, são o resultado de dois ovos fertilizados por dois espermatozoides e representam $2 / 3$ dos gemelares. Também são designados de bivitelinos, fraternos e biovulares. Em relação, a placentação é apresentada somente na forma DC, apesar da placenta poder ser fusionada (REZENDE JF; MONTENEGRO CA, 2014).

Na obstetrícia moderna, houve o aumento da prevalência de gestação gemelar, principalmente nas últimas décadas e das complicações materno-fetais associadas. Alguns dos fatores que podem ser considerados determinantes para esse aumento é a tendência de muitas mulheres em adiar a gestação para idades mais tardias. Além disso, é possível acrescentar a larga utilização de drogas para indução da ovulação e de técnicas em reprodução humana assistida (NORWITZ ER, et al., 2005; LEE YM, et al., 2006). 
A gestação de gêmeos compreende cerca de 95\% dos nascimentos múltiplos. Muitos nuances envolvem seu prognóstico e permanecem não bem definidos, provocando grande controvérsia sobre os resultados perinatais no nascimento de gêmeos. Assim, não é possível desconsiderar um número grande de eventos desfavoráveis que ocorrem nos frutos da concepção, decorrentes de causas fetais, obstétricas ou maternas, independentemente do desenvolvimento fetal esperado (JOSEPH KS, et al., 2003; SHINWELL ES e HAKLAI $\mathrm{T}$, 2009).

Portanto, se justifica a relevância desta pesquisa para a comunidade científica e para a população em geral, bem como para ser ferramenta de planejamento de projetos para atingir o bem-estar da gestante de gêmeos. Deste modo, o presente estudo teve como objetivo identificar as complicações em gestações gemelares e relacioná-las com as consequências materno-fetais.

\section{MÉTODOS}

Trata-se de uma revisão integrativa da literatura, que se baseia na síntese de variados estudos publicados em uma faixa de tempo e, possibilita resultados gerais acerca de uma particular área de estudo (BOTELHO LLR, et.al., 2011). Esse método segue passos seguidos como: a identificação da problemática, ou seja, a área de estudo pesquisada; a busca da literatura com a delimitação dos descritores, operadores booleanos e bases de dados segundo critério definidos para a seleção de cada artigo; a avaliação e análise dos dados obtidos em cada artigo, como mostrado na Figura 1. Sendo que cada artigo, busca-se responder à pergunta central da pesquisa em questão: Quais são as complicações da gestação de gemelaridade e quais consequências acarretam para a puérpera?

Figura 1 - Fluxograma com a Seleção da amostra final dos artigos.

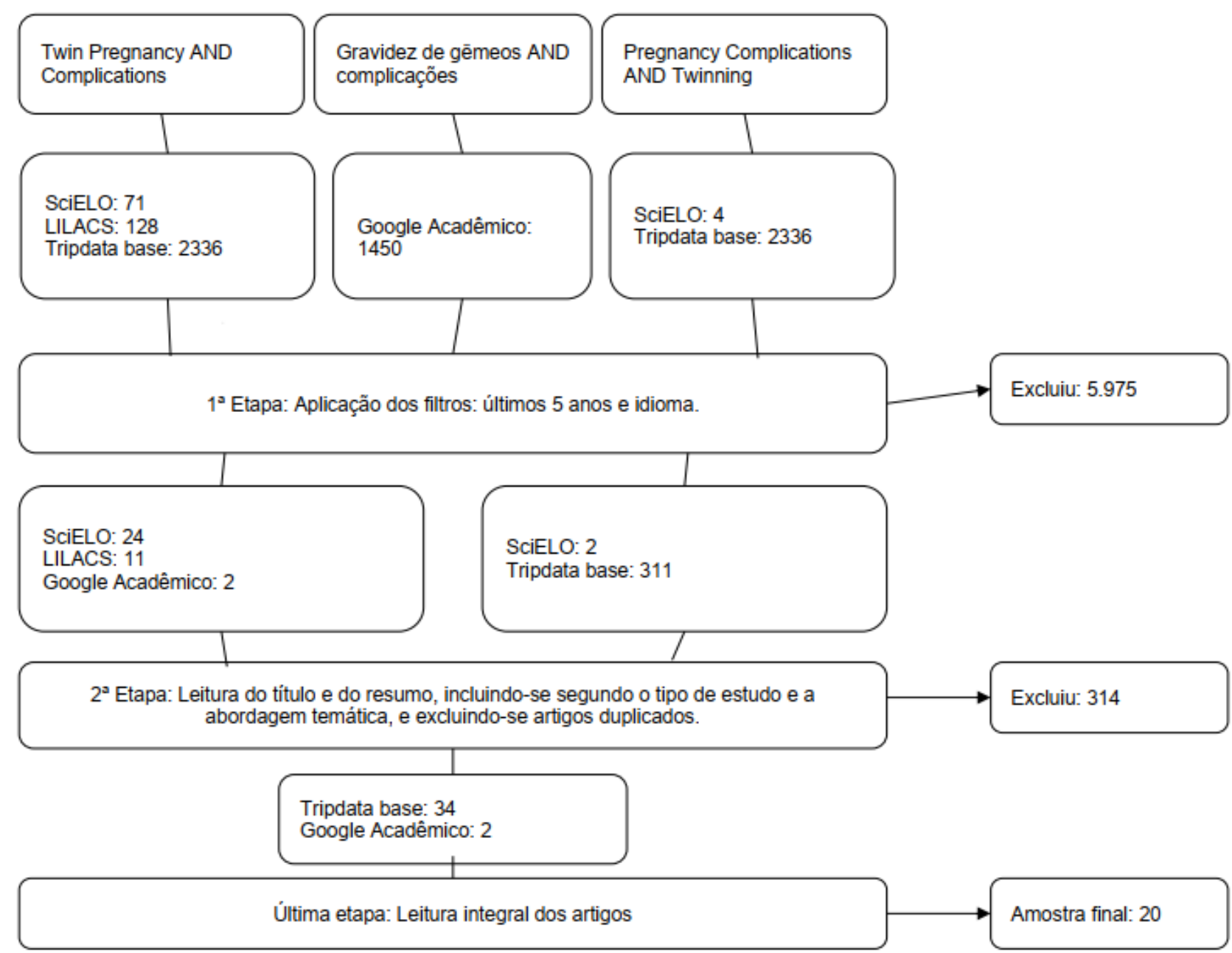

Fonte: Caçapava DXSC, et al., 2020 
A etapa de coleta de dados ocorreu a partir das plataformas Scientific Electronic Library Online (SciELO), Literatura Latino-Americana do Caribe em Ciências da Saúde (LILACS), Turning Research Into Practice (TRIP DATABASE) e Google Acadêmico, no mês de setembro de 2020. A busca foi realizada por meio dos Descritores em Ciência da Saúde (DeCS/MeSH), em português e em inglês: Gravidez de Gêmeos (Pregnancy, Twin), Complicações (Complications), Complicações na Gravidez (Pregnancy Complications), Gemelaridade Monozigótica (Twinning, Monozygotic), combinando-os com o booleano "AND", como Pregnancy complications AND twinning e Twin pregnancy AND complications. Dessa forma, a $1^{a}$ etapa deuse pela aplicação de filtros disponibilizados pelas bases de dados, como o período de publicação, sendo considerado os últimos cinco anos, além dos idiomas em português, inglês, francês e espanhol, a partir disso foram encontrados ao todo 350 artigos.

A etapa de seleção, $2^{\mathrm{a}}$ etapa, foi conduzida de acordo com a leitura do título e do resumo, utilizou-se os critérios de inclusão referentes ao tipo de estudo (estudo em coorte, estudos transversais e relato de caso, em última instância) e abordagem temática. Excluiu-se os artigos que estavam duplicados nos bancos de dados buscados, trabalhos científicos que não falassem sobre complicações maternas e/ou fetais em gestação gemelar e que não tratem dos aspectos éticos da pesquisa, chegando à totalidade de 34 artigos, com base na leitura dinâmica do título e dos resumos dos artigos.

$\mathrm{Na}$ última etapa, foi realizada a leitura exploratória dos artigos e então selecionados 20 artigos. Tais artigos foram escolhidos por apresentarem aspectos que respondiam à questão norteadora, e a análise do conteúdo permitiu a organização dos dados em categorias temáticas. Depois os dados foram sintetizados de maneira descritiva, possibilitando ao leitor a avaliação dos dados encontrados, em um quadro que contemplou os seguintes aspectos considerados essenciais: ano, autores, título e categoria pertencente.

\section{RESULTADOS}

As informações dos artigos foram extraídas e articuladas na Quadro 1, contendo o ano, nome dos autores, título e categoria pertencente, em relação às complicações maternas e fetais durante as gestações múltiplas. Dessa forma, os artigos selecionados correspondem aos anos de 2015 (4 artigos), 2016 (5 artigos), 2017 (4 artigos), 2018 (3 artigos), 2019 (4 artigos). No que diz respeito aos idiomas, tem-se que 4 artigos são em português, 1 em francês e 15 em inglês. Segundo os locais de elaboração, 4 artigos tiveram sua produção realizada no Brasil, 4 nos Estados Unidos da América, 2 em Portugal, 2 na França, 1 na Finlândia, 1 na Dinamarca, 2 na Irlanda, 1 na Itália, 2 no Reino Unido e 1 na Austrália. 
Quadro 1 - Quadro expositivo dos artigos selecionados, de acordo com autor, ano de publicação, título e categoria.

\begin{tabular}{|c|c|c|}
\hline $\begin{array}{l}\text { CATEGORIA } \\
\text { PERTENCENTE }\end{array}$ & TÍTULO & $\begin{array}{l}\text { AUTORES } \\
\text { E ANO }\end{array}$ \\
\hline Complicações fetais & Twin pregnancies complicated by gestational diabetes mellitus: a single centre cohort study & DINHAM GK, et al., 2016 \\
\hline Complicações fetais & $\begin{array}{l}\text { Une complication rare de la grossesse gémellaire monochoriale: la séquence Twinreversed arterial } \\
\text { perfusion (TRAP) }\end{array}$ & JAYI S, et al., 2015 \\
\hline Complicações fetais & Short- and long-term perinatal outcome in twin pregnancies affected by weight discordance & VEDEL C, et al., 2017 \\
\hline Complicações fetais & Absorção de gemelar na gravidez - relato de caso & ALVES A. P. P. F.; et al., 2019 \\
\hline Complicações maternas & Death of One Twin during the Perinatal Period: An Interpretative Phenomenological Analysis & $\begin{array}{l}\text { MEANEY S, CORCORAN P e } \\
\text { O'DONOGHUE K, } 2016\end{array}$ \\
\hline Complicações maternas & Twin Pregnancy and Severe Maternal Outcomes & SANTANA DS, et al., 2016 \\
\hline Complicações maternas & $\begin{array}{l}\text { Maternal functional hemodynamics in uncomplicated twin pregnancies: A longitudinal study using } \\
\text { impedance cardiography }\end{array}$ & ORABONA R, et al., 2018 \\
\hline Complicações maternas & Maternal complications in twin pregnancies in Finland during 1987-2014: a retrospective study & RISSANEN AS, et al., 2019 \\
\hline $\begin{array}{l}\text { Complicações maternas } \\
\text { e fetais }\end{array}$ & Mothers' perspectives on the perinatal loss of a co-twin: a qualitative & RICHARDS J, et al., 2015 \\
\hline $\begin{array}{l}\text { Complicações maternas } \\
\text { e fetais }\end{array}$ & $\begin{array}{l}\text { Adverse maternal outcomes and birth weight discordance in twin gestation: British Columbia, Canadian } \\
\text { data }\end{array}$ & JAHANFAR S e LIM K, 2017 \\
\hline $\begin{array}{l}\text { Complicações maternas } \\
\text { e fetais }\end{array}$ & $\begin{array}{l}\text { Gravidez gemelar monocoriônica biamniótica: experiência do Hospital de Santa Maria nos últimos } 5 \\
\text { anos. }\end{array}$ & NEVES NM, 2017 \\
\hline $\begin{array}{l}\text { Complicações maternas } \\
\text { e fetais }\end{array}$ & Impact of oocyte donation on perinatal outcome in twin pregnancies & GUILBAUD L, et al., 2017 \\
\hline $\begin{array}{l}\text { Complicações maternas } \\
\text { e fetais }\end{array}$ & Restrição de crescimento seletiva em gestação gemelar monocoriônica: relato de caso & FILHO PWLL, et al., 2015 \\
\hline $\begin{array}{l}\text { Complicações maternas } \\
\text { e fetais }\end{array}$ & $\begin{array}{l}\text { Association between hypertensive disorders and fetal growth restriction in twin compared with singleton } \\
\text { gestations }\end{array}$ & PROCTOR LK, et al., 2018 \\
\hline $\begin{array}{l}\text { Complicações maternas } \\
\text { e fetais }\end{array}$ & Induction of twin pregnancy and the risk of caesarean delivery: a cohort study & JONSSON M, 2015 \\
\hline $\begin{array}{l}\text { Complicações maternas } \\
\text { e fetais }\end{array}$ & $\begin{array}{l}\text { Perinatal outcomes in twin pregnancies complicated by maternal morbidity: evidence from the WHO } \\
\text { Multicountry Survey on Maternal and Newborn Health }\end{array}$ & SANTANA DS, et al.; 2018 \\
\hline $\begin{array}{l}\text { Complicações maternas } \\
\text { e fetais }\end{array}$ & Monochorionic versus dichorionic twins: Are obstetric outcomes always different? & NUNES FC, et al., 2016 \\
\hline $\begin{array}{l}\text { Complicações maternas } \\
\text { e fetais }\end{array}$ & Síndrome de Transfusão Feto-Fetal & RODRIGUES APPS, 2016 \\
\hline $\begin{array}{l}\text { Complicações maternas } \\
\text { e fetais }\end{array}$ & Hypertensive disorders of pregnancy in twin gestations complicated by gestational diabetes & MOURAD M, et al., 2019 \\
\hline $\begin{array}{l}\text { Complicações maternas } \\
\text { e fetais }\end{array}$ & $\begin{array}{l}\text { The maternal and perinatal implications of hypertensive disorders of pregnancy in a multiple pregnancy } \\
\text { cohort }\end{array}$ & HAYES-RYAN D, et al., 2019 \\
\hline
\end{tabular}

Fonte: Caçapava DXSC, et al., 2020. 
A partir da avaliação dos dados foi possível elencar duas categorias globais, uma referente às complicações fetais e outra às complicações maternas. Dessa forma, como complicações fetais, tem-se o aborto precoce, aborto tardio, morte fetal (após 22 semanas), anomalias fetais, crescimento discordante, síndrome de transfusão feto-fetal (STFF), reversão da perfusão arterial dos gêmeos/acardia fetal (R.P.A.G), morte de um dos fetos, pequeno para a idade gestacional (PIG), necessidade de internação e baixo peso. As complicações maternas encontradas foram distúrbios hipertensivos, pré-eclampsia, diabetes gestacional, parto prematuro, rupturas prematuras (RPM) de grau I, II e III, hemorragia pós-parto, rotura de placenta, luto após perda de um gêmeo, sepse e morte materna.

Por intermédio de uma análise quantitativa da frequência de citação de cada complicação nos artigos incluídos, e utilizando os recursos gráficos disponibilizados pela plataforma de formulários do Google, foi possível estratificar a incidência de cada complicação, seja ela fetal ou materna. Os resultados encontrados foram: complicações maternas - parto prematuro (50\%), distúrbios hipertensivos (43,8\%), pré-eclampsia e eclampsia $(37,5 \%)$, ruptura prematura das membranas $(37,5 \%)$, hemorragia pós-parto $(31,3 \%)$, diabetes gestacional $(25 \%)$, rotura de placenta $(18,8 \%)$, morte materna $(12,6 \%)$, luto após perda de um dos gêmeos $(12,5 \%)$, colestase obstétrica $(6,3 \%)$, sepse $(6,3 \%)$, síndrome HELLP $(6,3 \%)$ e parto cesáreo $(6,3 \%)$; complicações fetais - crescimento discordante $(53,3 \%)$, baixo peso $(46,7 \%)$, morte fetal (após 22 semanas) $(33,3 \%)$, anomalias fetais $(13,3 \%)$, síndrome de transfusão feto-fetal $(13,3 \%)$, internação $(13,3 \%)$, aborto precoce $(6,7 \%)$, aborto tardio $(6,7 \%)$, apneia do recém-nascido $(6,7 \%)$, hipoglicemia $(6,7 \%)$, hemorragia intraventricular $(6,7 \%)$, pequeno para idade gestacional $(6,7 \%)$, reversão da perfusão arterial $(6,7 \%)$, admitidos na UTI neonatal (6,7\%), morte de um dos fetos (6,7\%). Além disso, é importante ressaltar que muitas complicações não ocorrem isoladamente, assim, explica a somatória das porcentagens não fecharem em $100 \%$.

Outro aspecto detectado nas análises dos artigos, foram as relações entre as complicações maternas e as complicações fetais. Foram identificados vínculos de causa, consequência e adição no progresso e prognóstico das complicações. As relações de causa encontradas foram entre ruptura prematura de membrana - parto prematuro, distúrbios hipertensivos -, parto cesáreo e crescimento discordante - parto prematuro. Já as consequências constatadas foram a associação entre eclampsia - morte materna, anomalias fetais - aborto precoce, distúrbios hipertensivos - parto prematuro, STFF - morte fetal, anormalidades fetais luto pós parto e STFF - parto prematuro, e, por fim, os fatores de adição são diabetes gestacional - distúrbios hipertensivos, STFF - anomalias fetais, baixo peso - crescimento discordante e hemorragia pós-parto - sepse.

\section{DISCUSSÃO}

\section{Complicações maternas}

As complicações maternas mais recorrentes identificadas foram o parto prematuro, distúrbios hipertensivos, hemorragia pós-parto, pré-eclampsia, eclampsia, diabetes gestacional e ruptura prematura das membranas. Além disso, há complicações menos incidentes como síndrome HELLP (caracterizada por hemólise, enzimas hepáticas elevadas, baixa contagem de plaquetas, além de não ser restrita a gravidez gemelar), sepse, mortalidade materna, colestase obstétrica, além do luto após a perda de um gêmeo. Desse modo, foram abordadas as complicações maternas mais frequentes em relação a gestação gemelar, sendo que a maioria resulta em morte materna e outros problemas (SANTANA DS, et al., 2016).

A ruptura prematura de membranas (RPM), segundo o Boletim Prático do Colégio Americano de Ginecologia e Obstetrícia, é a perda de líquido amniótico antes do início do trabalho de parto. Em relação a RPM, houve uma prevalência de partos espontâneos em relação aos partos induzidos por ocitocina (JONSSON M, 2015). Portanto, é possível associar a ruptura prematura de membranas com o aumento de casos de partos prematuros em gestações gemelares (RISSANEN AS et al., 2019; NUNES FC, et al., 2016; PROCTOR LK, et al., 2018; SANTANA DS, et al., 2016; RODRIGUES APPS, 2016).

O parto prematuro ( $<37$ semanas) foi prevalente na literatura e decorrente das combinações de nuliparidade, idade avança, crescimento fetal discordante e fatores de risco, por exemplo, tabagismo ou obesidade, como afirmam Rissanem AS, et al (2019), Jahanfar S e Lim K (2017) e colaboradores. Também, 
houve uma maior taxa de nascimento prematuro em gestações monocoriônicas diamnióticas, segundo Nunes FC, et al. (2016) e colaboradores. Outra condição associada à parto prematuro, segundo Rodrigues APPS (2016), foi a Síndrome de transfusão feto-fetal, sendo que essa ocorre em 8-10\% das gestações monocoriônicas. Além disso, no estudo do Proctor LK, et al. (2018) e associados e na pesquisa de HayesRyan D, et al. (2019) e parceiros, houve uma relação estreita entre gestações com distúrbios hipertensivos e a prematuridade, em que houve 2 vezes mais chance de parto prematuro.

Além de sua relação com a prematuridade, os distúrbios hipertensivos apresentaram incidência significativa nas gestações gemelares. Dentre os riscos de desenvolvimento de distúrbios hipertensivos descritos no estudo de Hayes-Ryan D, et al. (2019), cita-se a idade materna maior que 40 anos, nuliparidade, a concepção por meio de oócito doados e a presença de colestase obstétrica. Ademais, de acordo com Mourad M, et al. (2019), a presença de diabetes mellitus gestacional apresentou-se, inicialmente, como um risco para o desenvolvimento de distúrbios hipertensivos, no entanto, após o ajuste da idade materna, raça, o uso de fertilização in vitro e o índice de massa corporal na gravidez, tal relação com a presença de diabetes perdeu significância. A presença de diabetes mellitus gestacional também foi observada em Hayes-Ryan $D$, et al. (2019), no qual ele concorda com Mourad M, et al. (2019), que o diagnóstico de tal patologia não aumenta a probabilidade de desenvolvimento de distúrbios hipertensivos.

As funções hemodinâmicas maternas também são alteradas durante a gestação de gêmeos. Observouse oscilações do débito cardíaco ao longo das semanas gestacionais, sendo que em 34 semanas houve uma redução significativa desse. Além disso, após metade da gestação gemelar foi encontrada uma reduzida reserva de pré carga, cujo qual é um mecanismo fisiológico a fim de compensar a hipervolemia durante o trabalho de parto, impedindo a ocorrência de hemorragia (ORABONA R, et al., 2019).

O luto após a perda de um gêmeo, consequência materna prevalente em todas as gestações com desfechos negativos, é um choque profundo vivido pelos pais, que provoca impacto durante toda a vida do casal. (MEANEY S, et al., 2016). A análise conduzida por Meaney S, et al (2016), a partir do diagnóstico prénatal de anormalidade fetal de grande mortalidade, evidenciou o total despreparo dos pais para lidar com a descoberta e com o futuro prognóstico. Houve também uma tentativa pelos profissionais de saúde para mudança de foco da experiência traumática dos pais para o cuidado e para a concentração ao bebê sobrevivente, sugerindo, portanto, um adiamento do luto por meses ou anos, como evidencia Richards $\mathrm{J}$, et al. (2015). Dessa maneira, torna-se fundamental, segundo Richards J, et al. (2015), muitos serviços de apoio psicológico para lidar com as necessidades complexas que acometem os pais que perdem um filho.

A pré-eclampsia é caracterizada pelo aumento da pressão arterial associada ao aumento de proteínas na urina, já a eclampsia é identificada como convulsões durante o parto, ou seja, consequência da hipertensão e proteinúria durante a gravidez (Guidelines, 2015). Esse tipo de complicação na gravidez gemelar é recorrente (JONSSON M, 2015; GUILBAUD L, et al., 2017; PROCTOR LK, et al., 2018 e SANTANA DS, et al., 2016). Devido a isso, o estudo de Johnsson M (2015) comprova a relação entre a pré-eclampsia e eclampsia à indução do parto e consequentemente aos partos pré-termo. No entanto, o artigo de Proctor LK, et al. (2018) afirmou ser conflitante os dados para fazer a associação entre a pré-eclâmpsia e o crescimento fetal em gestação gemelar, mas em gestações únicas é comprovada essa relação. Dessa forma, é importante uma maior atenção clínica a essas gestantes, pois a pré-eclâmpsia e a eclampsia estão associadas a um maior risco de morte materna (SANTANA DS, et al., 2016).

\section{Complicações fetais}

As complicações fetais mais recorrentes são o crescimento discordante, baixo peso ao nascer e morte fetal. Além dessas, existem outras complicações que são encontradas em gestações gemelares e apresentam quadro clínico grave, como a internação, que está relacionada com apneia do $\mathrm{RN}$ e hipoglicemia, anomalia fetal e a Síndrome de Transfusão Feto-Fetal (STFF), de acordo com a análise dos artigos e livros usados nesta revisão integrativa.

A partir da análise das amostras literárias que integram o estudo, nota-se que o crescimento discordante foi frequentemente associado às complicações fetais. A restrição do crescimento fetal está vinculada às gestações em que um dos gêmeos apresenta padrão de crescimento abaixo do percentil 10 enquanto no 
outro o padrão é considerado apropriado (FILHO PWLL, et al., 2015). No que diz respeito às causas e repercussões, tem-se o fornecimento desigual de nutrientes para os fetos, devido, por exemplo, a anormalidades na inserção do cordão, divisão da placenta e inclusive complicações maternas, como préeclâmpsia e diabetes, esses fatores podem corroborar com o desenvolvimento de outros problemas para gestante, além da indução do trabalho de parto prematuro e necessidade de parto cesáreo (JAHANFAR, $\mathrm{S}$ e LIM K, 2017; JONSSON M, 2015; PROCTOR LK, et al., 2018; ALVES APPF, et al., 2019; NEVES NM, 2017; SANTANA DS, et al., 2016). Nesse viés, os resultados negativos manifestaram-se principalmente em gestações monocoriônicas diamnióticas (NUNES FC, et al., 2016).

O baixo peso $(1500-2500 \mathrm{~g})$ e muito baixo peso $(<1500 \mathrm{~g})$ também foram descritos como episódios frequentes em gestações gemelares, sendo correlacionados com o crescimento discordante, prematuridade e hipertensão materna (NEVES NM, 2017; FILHO PWLL, et al., 2015; NUNES FC, et al., 2016; PROCTOR LK, et al., 2018; HAYES-RYAN D, et al., 2019; SANTANA DS, et al., 2018). Outra eventualidade que pode levar ao quadro correspondente, é a Síndrome de Transfusão Feto-Fetal (STFF) que consiste em modificações hemodinâmicas fetais recorrentes em cerca de $10 \%$ das gestações monocoriônicas diamnióticas e provocam divergência no suprimento volêmico dos fetos, além de possuir potencial letal. Ademais, é notório que a anastomose arteriovenosa é considerada preditor da STFF (RODRIGUES APPS, 2016).

A morte fetal também é retratada com uma complicação fetal, gemelar, tanto monocoriônica quanto dicoriônica, porém pesquisas, entre os anos de 2010 e 2013, mostram que no caso da gravidez monocoriônica há quatro vezes mais probabilidade de ocorrer esse resultado adverso (DINHAM GK, et al., 2016). Outra pesquisa associou o aumento de casos de morte fetal em gestações gemelares que não foram resultado de uma fertilização in vitro (GUILBAUD L, et al., 2017).

A STFF também esteve atrelada à morte fetal, principalmente, a gestação monocoriônica diamniótica, em que cerca de $50 \%$ dos gêmeos sobreviventes também morreram ou desenvolveram déficit neurológico. (RODRIGUES APPS, 2016). Em uma análise retrospectiva, no Hospital de Santa Maria em Portugal, das gravidezes gemelares ficou evidente a associação entre patologias graves, como a STFF, à mortalidade fetal, presente em $20 \%$ das gestações (NEVES NM, 2017).

Como visto na amostra deste estudo, há várias complicações maternas e fetais, no entanto poucas são detalhadas na literatura, por exemplo os distúrbios hipertensivos são recorrentes e discutidos em $43,8 \%$ dos artigos, porém não há uma explicação da causa desses distúrbios, limitando a presente discussão. Além disso, há uma falta de estudos quanto às complicações menos citadas como aborto tardio e precoce, reversão da perfusão arterial dos gêmeos, morte de um dos fetos, apneia do RN, hipoglicemia fetal, síndrome HELLP, sepse e morte materna. Apesar das limitações, ficou evidente que a gravidez gemelar possui maior probabilidade de apresentar complicações.

\section{CONSIDERAÇÕES FINAIS}

A análise das produções científicas encontradas nos permitiu concluir que há uma maior prevalência de complicações maternas e fetais em gestações gemelares além de taxas elevadas de complicações comparado a gestações únicas. Foram identificadas diversas complicações, desenvolvidas ao longo do estudo, separadas em duas categorias: maternas e fetais, que embora estejam separadas didaticamente, a maioria das complicações se relacionam, sendo algumas associações entre causa e consequência, como, por exemplo, a eclampsia que pode levar a morte materna, e a discordância de crescimento entre os gêmeos que pode levar ao parto prematuro. Concluímos que por mais que a morte materna não seja uma complicação recorrente a maioria das outras complicações maternas podem ter como consequência o óbito da gestante.

\section{AGRADECIMENTOS}

Agradecemos o curso de Medicina do Centro Universitário de Anápolis - UniEVANGÉLICA pela oportunidade e suporte durante a elaboração desse trabalho. 


\section{REFERÊNCIAS}

1. ALVES APPF, et al. Absorção de gemelar na gravidez, Brasil. Anais da Faculdade de Medicina de Olinda, 2019; 1(3).

2. BOTELHO LLR, et.al. O método da revisão integrativa nos estudos organizacionais. Gestão e Sociedade, 2011; 11(5): 121-136.

3. DINHAM GK, et al. Twin pregnancies complicated by gestational diabetes mellitus: a single centre cohort study. Diabetic medicine: a journal of the British Diabetic Association, 2016; 33(12): 1659-1667.

4. FILHO PWLL, et al. Restrição de crescimento seletiva em gestação gemelar monocoriônica: relato de caso. Rev Med UFC, 2015; 1(55): 57-60.

5. GUILBAUD L, et al. Impact of oocyte donation on perinatal outcome in twin pregnancies. 7 American Society for Reproductive Medicine, 2017; 107 (4): 948-953.

6. HAYES-RYAN D, et al. The maternal and perinatal implications of hypertensive disorders of pregnancy in a multiple pregnancy cohort. Acta Obstet Gynecol Scand, 2020; 99(4): 525-536.

7. JAHANFAR S, LIM K. Adverse maternal outcomes and birth weight discordance in twin gestation: British Columbia, Canadian data. International Journal of Women's Health, 2017; 9: 871-878.

8. JAYI S, et al. Une complication rare de la grossesse gémellaire monochoriale: la séquence Twin-reversed arterial perfusion (TRAP). The Pan African Medical Journal, 2015; 20 (1); 347.

9. JONSSON, M. Induction of twin pregnancy and the risk of caesarean delivery: a cohort study. Department of Women's and Children's Health, 2015, 15 (136): 1-7.

10. JOSEPH, K, et al. A parsimonious explanation for intersecting perinatal mortality curves: understanding the effect of plurality and of parity. BMC pregnancy and childbirth, 2003;3(1): 3.

11. LEE YM, et al. The impact of multiple gestations on late preterm (near-term) births. Clin Perinatol, 2006; 33(4):777- 92.

12. MEANEY S, et al. Death of One Twin during the Perinatal Period: An Interpretative Phenomenological Analysis. Journal of palliative medicine, 2017; 20(3): 290-293.

13. MOURAD M, et al. Hypertensive disorders of pregnancy in twin gestations complicated by gestational diabetes. The Journal of Maternal-Fetal \& Neonatal Medicine, 2019; (16): 1-5.

14. NEVES NM. Gravidez gemelar monocoriônica biamniótica: experiência do Hospital de Santa Maria nos últimos 5 anos. Tese (Mestrado Integrado em Medicina) - Clínica Universitária de Ginecologia e Obstetrícia do Hospital de Santa Maria, Universidade de Lisboa. Lisboa, 2016, p. 1-14.

15. NORWITZ ER, et al. Maternal Physiology and Complications of Multiple Pregnancy. Semin Perinatol, 2005; 29:338348.

16. NUNES FC, et al. Monochorionic versus dichorionic twins: Are obstetric outcomes always different? Journal of Obstetrics and Gynaecology, 2016; 5(36): 598-601.

17. ORABONA R, et al. Maternal functional hemodynamics in uncomplicated twin pregnancies: A longitudinal study using impedance cardiography. Acta Obstet Gynecol Scand, 2019; 2(98): 188-195.

18. PROCTOR LK, et al. Association between hypertensive disorders and fetal growth restriction in twin compared with singleton gestations. American Journal of Obstetrics \& Gynecology, 2019; 221 (3): 251.e1-251.e8

19. REZENDE JF, MONTENEGRO CAB. Rezende Obstetrícia Fundamental. 13.ed. Rio de Janeiro, Guanabara Koogan, 2014).

20. RICHARDS J, et al. Mothers' perspectives on the perinatal loss of a co-twin: a qualitative study. BMC Pregnancy Childbirth, 2015; Jul 2; 15(143).

21. RISSANEN AS, et al. Maternal complications in twin pregnancies in Finland during 1987-2014: a retrospective study. BMC Pregnancy and Childbirth, 2019: 19(337).

22. RODRIGUES APPS. Síndrome De Transfusão Feto-fetal. Tese (Mestrado Integrado em Medicina) - Clínica Universitária de Ginecologia e Obstetrícia do Hospital de Santa Maria, Universidade de Lisboa. Lisboa, 2016, p. 3-16.

23. SANTANA DS, et al. Twin pregnancy and several maternal outcomes. Obstetrics \& Gynecology, 2016; 127(4).

24. SANTANA DS, et al. Perinatal outcomes in twin pregnancies complicated by maternal morbidity: evidence from the WHO Multicountry Survey on Maternal and Newborn Health. BMC Pregnancy and Childbirth, 2018; 449(18), 1-11.

25. SHINWELL ES, et al. Outcomes of multiplets. Neonatology, 2009; 95(1): 6-14.

26. SOCIEDAD ESPAÑOLA DE GINECOLOGÍA E OBSTETRICIA. Embarazo gemelar bicorial - Guia de Asistencia Práctica, Espanha. Revista Oficial de la Sociedad Española de Ginecología y Obstetricia. v. 59, № 1, p. 43-57, 2016.

27. VEDEL, C, et al. Short- and long-term perinatal outcome in twin pregnancies affected by weight discordance. Acta obstetricia et gynecologica Scandinavica, 2017; 96(2): 233-242.

28. YANG F, et al. High risk twin pregnancy complicated with severe rachiterata and huge dorsal mass suffering from refractory infection-A case report. Journal Medicine, 2019; 98 (11); e14462. 